\title{
Trabajo cooperativo en el aula de fundamentos de teoría de máquinas y mecanismos
}

\author{
Juan José Prieto-Romero ${ }^{a} \&$ María Ángeles Martín-Maraver ${ }^{b}$ \\ ${ }^{a}$ Escuela Superior de Ingeniería, Universidad de Huelva, Huelva, España. juan.prieto@dimme.uhu.es \\ ${ }^{b}$ Centro del Profesorado Sevilla 6, Lora del Río, Sevilla, España.mariangeles@loracep.org
}

\begin{abstract}
Resumen-El artículo desarrolla una propuesta de innovación docente, en el contexto universitario, con el objetivo de mejorar el nivel de formación de los estudiantes y el rendimiento académico en la asignatura de Fundamentos de Teoría de Máquinas y Mecanismos (F.T.M.M.). Teniendo en cuenta estudios y experiencias que se comparten entre la comunidad educativa, se derivó la necesidad de pasar de la clase magistral a una clase que propiciara el protagonismo del estudiante en relación a su propio aprendizaje. Se decidió implementar en el aula técnicas simples de trabajo cooperativo, porque, de una forma sencilla y sin cambios metodológicos profundos, permitía al docente pasar de un modelo tradicional, centrado en la enseñanza, a un modelo centrado en el aprendizaje. El resultado fue el impulso de la participación activa y autónoma del alumnado y la mejora de sus competencias, su capacitación y su cualificación. En definitiva, las técnicas de trabajo cooperativo se mostraron eficaces en la mejora del rendimiento académico del alumnado.
\end{abstract}

Palabras Clave - Trabajo cooperativo, metodologías activas, competencias, aprendizaje autónomo.

Recibido: 6 de julio de 2016. Revisado: 16 de agosto de 2016. Aceptado: 14 de septiembre de 2016.

\section{Cooperative work in the classroom of fundamentals of machine and mechanism theory}

\begin{abstract}
The article develops a proposal for teaching innovation in the university context, with the aim of improving the level of training of students and academic performance in the subject Fundamentals of Theory of Machines and Mechanisms (F.T.M.M.). Given studies and experiences shared among the educational community, was derived the need to move from a master class to a class that encouraged student leadership in relation to their own learning. It was decided to implement, in classroom, simple techniques cooperative work, because, in a simple way and without deep methodological changes, allowed teachers to move from a traditional model, focused on teaching, to a learningcentered model. The result was the impetus of the active and autonomous participation of students and improving their skills, training and qualifications In short, cooperative work techniques were effective in improving the academic performance of students.
\end{abstract}

Keywords - Cooperative work, active methodologies, skills, autonomous learning.

\section{Introducción}

Vivimos en un mundo global que se caracteriza por un incesante cambio. El ritmo de producción del conocimiento es cada vez más extenso y acelerado y, por tanto, su obsolescencia también crece. Estamos educando para un futuro incierto en el que nuestro alumnado trabajará en profesiones que aún no existen. Por ello, nuestros estudiantes deben prepararse para incorporarse a un entorno laboral muy diferente al que existía hace solo diez años atrás. Los problemas que estos futuros profesionales deberán enfrentar cruzan las fronteras de las disciplinas y demandan enfoques innovadores y habilidades para la resolución de problemas complejos. En esta sociedad del conocimiento el docente ya no es el depositario y transmisor esencial del saber. Ante esta perspectiva educativa, ¿cómo ha de enfocarse la Universidad al objeto de hacerla realmente eficaz y competitiva?

Para integrar la Universidad en la sociedad del conocimiento, 29 Ministros Europeos de Educación firmaron en Bolonia, el 19 de Junio de 1999, la Declaración de Bolonia para reformar las estructuras de los sistemas de educación superior de manera convergente. Se trataba, no solo de un precepto político, sino también un compromiso fijo a un programa de acción destinado a reformar el sistema de educación superior en los países europeos, cuyo principal objetivo consistía en la creación de un Espacio Europeo de Educación Superior (EEES) que facilitara que los títulos europeos tuviesen reconocimiento en toda Europa, mejorando la contratación y la movilidad de los futuros profesionales.

Un aspecto importante del EEES es que implica la instauración de nuevas metodologías docentes, en detrimento de las tradicionales clases magistrales. Los cambios en el perfil docente están basados en una docencia centrada en el alumnado, lo que convierte al profesorado en guía y facilitador del aprendizaje autónomo del estudiante. Se establece también la necesidad de promover un nuevo enfoque basado en la adquisición de competencias del discente ya que, en el proceso de aprendizaje, se elimina la división entre teoría y práctica al requerirse el análisis y resolución de problemas unidos a la búsqueda de alternativas frente a las situaciones que plantean dichos problemas. Es decir, el aprendizaje está orientado a que se adquieran conocimientos teóricos, habilidades, destrezas, la capacidad de trabajar en equipos multidisciplinarios y la facultad de aprender a aprender. Desde esta perspectiva, el

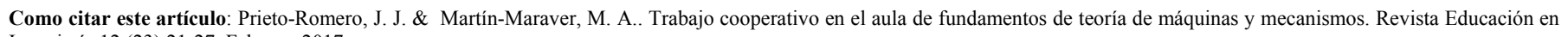
Ingeniería 12 (23) 21-27, Febrero, 2017. 
concepto de competencia en la educación se interpreta como saberes de ejecución.

Este enfoque exige pasar de la clase magistral a una clase que facilite el protagonismo del estudiante en relación a su propio aprendizaje. En consecuencia, el docente necesita experimentar metodologías que pasen de un modelo tradicional centrado en la enseñanza, a un modelo centrado en el aprendizaje. Esos modelos activos facilitan la participación activa y autónoma del alumnado, quien ha de implicarse y asumir la responsabilidad de su aprendizaje. Dar respuesta a estas exigencias constituye el origen de aplicar técnicas de trabajo cooperativo en la asignatura de Fundamentos de Teoría de Máquinas y Mecanismos (F.T.M.M.).

Fundamentos de Teoría de Máquinas y Mecanismos busca comprender las relaciones entre la geometría y los movimientos de las piezas de una máquina o mecanismo y las fuerzas actuantes. Dentro de la F.T.M.M. se distingue entre la síntesis o diseño y el análisis. Síntesis o diseño es el proceso creativo mediante el cual es posible generar un modelo o patrón, para satisfacer una necesidad, cumpliendo las restricciones cinemáticas y dinámicas que definen el problema. Es decir, partiendo de una necesidad, diseñamos un mecanismo. Análisis es el estudio del comportamiento de un mecanismo ya conocido, con el fin de determinar si es adecuado para el trabajo al que está destinado.

Mediante la asignatura F.T.M.M. se aplica el conocimiento científico a problemas prácticos. Resolver un problema implica realizar tareas que demandan procesos de razonamientos más o menos complejos y no simplemente una actividad asociativa y rutinaria. Para desarrollar esta competencia se ha de fomentar la confianza del alumno en su propio pensamiento, potenciar las habilidades y capacidades para aprender, comprender y aplicar los conocimientos y favorecer la autonomía intelectual que le permita continuar su proceso de formación. También contribuye al desarrollo de otras competencias básicas como el trabajo en equipo, la creatividad, el análisis o el liderazgo.

Para los objetivos de bajo nivel, por ejemplo la adquisición y compresión de la información, cualquier método puede ser adecuado. Sin embargo, para los objetivos superiores como el desarrollo del pensamiento crítico y aprendizaje autónomo propios de la F.T.M.M. los métodos centrados en los alumnos son más eficaces. Por ello, se toma la opción de investigar, llegando a la decisión de profundizar en el trabajo cooperativo.

El Aprendizaje Cooperativo es un término genérico usado para referirse a un grupo de procedimientos de enseñanza que parten de la organización de la clase en pequeños grupos mixtos y heterogéneos donde los alumnos trabajan conjuntamente de forma coordinada entre sí para resolver tareas académicas y profundizar en su propio aprendizaje. En esta situación de aprendizaje los objetivos de los participantes se hallan estrechamente vinculados, de tal manera que cada uno de ellos sólo puede alcanzar sus objetivos si y sólo si los demás consiguen alcanzar los suyos [1]. El Aprendizaje Cooperativo establece un sistema de interacciones cuidadosamente diseñado que organiza e induce la influencia recíproca entre los integrantes de un equipo.

El aula cooperativa se fundamenta en los postulados de la teoría sociocultural de Vygotsky, la teoría genética de Piaget y su desarrollo a través de la Escuela de Psicología Social de Ginebra, la teoría de la Interdependencia Positiva de los hermanos Johnson, el aprendizaje Significativo de Ausubel, la Psicología Humanista de Rogers y la Teoría de las Inteligencias Múltiples de Gardner.

¿Por qué implementar el trabajo cooperativo en el aula? Acorde con Pierre Dillembourgh [2], aplicar el trabajo cooperativo en el aula tiene como base el hecho de entender que los individuos no aprenden porque ellos sean individuales o porque estén en grupo, sino porque ejecutan algunas actividades (leer, predecir, etc.) que conllevan algunos mecanismos de aprendizaje (inducción, predicción, compilación, etc). Pero además, la interacción entre sujetos genera actividades adicionales (explicación, regulaciones mutuas, etc.). En este sentido, el aprendizaje cooperativo desarrolla competencias no solo referidas a los contenidos, sino también orientadas al desarrollo de habilidades y destrezas individuales (planificación, seguimiento, evaluación...) e interpersonales (cooperación, liderazgo, responsabilidad, compromiso), con claros beneficios para el aprendizaje de los alumnos.

Con este planteamiento, se establece la hipótesis de que las dificultades a las que se enfrentan los estudiantes ante la resolución de los problemas de Fundamentos de Teoría de Máquinas y Mecanismos podrían minimizarse con el apoyo y tutorización entre iguales. Se decide, por tanto, desarrollar una experiencia de trabajo en equipo que suponga la búsqueda de la implicación de todos los miembros $\mathrm{y}$, al buscar el éxito del grupo, lo más adecuado es utilizar técnicas de trabajo cooperativo.

\section{Metodología}

Teniendo en cuenta las recomendaciones del Servicio de Innovación Educativa de la Universidad Politécnica de Madrid acerca de cómo implementar en el aula una técnica cooperativa, que favoreciera el trabajo por competencias, se hizo necesario organizar detenidamente todo el proceso [3]. Para ello, siguiendo a Leonor Prieto Navarro [4], profesora en el Departamento de Didáctica y Teoría de la Educación de la Universidad Autónoma de Madrid, se establecieron las siguientes fases:

\subsection{Fase I: Planificación}

El objetivo era afianzar la capacidad del alumnado en la resolución de problemas de análisis cinemático. La tarea del alumnado sería, no solo buscar la solución del problema planteado, sino evidenciar las dudas y resolverlas entre los propios estudiantes.

Se decidió partir de un trabajo individual inicial previo e ir confrontando los resultados obtenidos entre el alumnado. La técnica simple 1-2-4 desarrolla agrupaciones progresivas, lo que permitía organizar el trabajo desde lo individual hasta el gran grupo de manera escalonada. Esta técnica consiste en que cada alumno/a plantea de manera individual la resolución del problema. Posteriormente, se agrupan en parejas, comparan sus respuestas y sintetizan una solución en conjunto. Se vuelven a unir de cuatro en cuatro (y de ocho en ocho si fuese un grupo grande) con el mismo objetivo. Estos últimos equipos han de elegir un 
representante que explicará al resto de la clase la respuesta elaborada por su grupo. El profesor guía esas explicaciones, especialmente buscando aclarar las dudas y aquellas dificultades que impiden la adecuada resolución del problema.

No se dejó al azar los distintos agrupamientos. Era necesario no solo favorecer la diversidad de interacciones entre todo el alumnado del aula, sino también evitar el enquistamiento de grupos ya establecidos y, muy importante, eliminar la posibilidad de que se diesen casos de estudiantes desubicados. Partiendo de la asignación de un número "n", del 1 al 30, de todos los estudiantes, se establecieron los siguientes criterios de agrupamientos:

a) Parejas: se agruparon los números pares e impares siguiendo un orden ascendente; es decir, el 1 y 3,5 y $7 \ldots 2$ y 4,6 y $8 \ldots$

b) Grupos de cuatro: se agruparon parejas pares e impares siguiendo un orden ascendente: $1 / 3 / 5 / 7,2 / 4 / 6 / 8 \ldots$ Restaron los estudiantes numerados como 29 y 30 que se distribuyeron en uno de los grupos impares y pares respectivamente.

c) Grupos de ocho: En esta última agrupación se unieron los cuartetos pares e impares consecutivos; es decir, 1/3/5/7/2/4/6/8 Programar los tiempos fue otro aspecto a tener en cuenta. Se decidió repartirlo como sigue:

a) Trabajo individual: 20 minutos.

b) Parejas: 10 minutos

c) Grupos de cuatro: 5 minutos

d) Grupos de ocho: 5 minutos.

Finalmente se estableció el aula como espacio donde transcurriría la actividad, ya que la disposición del mobiliario permitía la movilidad y agrupación del alumnado. En cuanto a las responsabilidades, de manera individual debían intentar dar resolución al problema planteado. En grupo tenían que compartir los resultados obtenidos, plantear e intentar resolver dudas, escuchar y respetar las intervenciones de los compañeros. Finalmente, en la última agrupación de 8 miembros se debía elegir un portavoz que resumiese el trabajo realizado y lo expusiera al resto de la clase.

Para valorar la cantidad y calidad de aprendizaje se estableció una evaluación cuantitativa y cualitativa de la experiencia. Por un lado, se pidió al alumnado que emitiese una calificación del 1 al 10 , teniendo en cuenta cómo la actividad colaborativa favorecía o no la resolución del problema y cómo permitía clarificar dudas y afianzar conocimientos. Por otro lado, la valoración cualitativa se desarrolló mediante la redacción de un texto individual en el que se debían expresar las impresiones acerca de la práctica colaborativa. También, se consideró importante que el docente proporcionase un cierre a la actividad emitiendo un resumen del trabajo que habían realizado los alumnos. En esta última fase se consideró muy importante evaluar el aprendizaje y los procesos de trabajo en equipo de los estudiantes.

\subsection{Fase II: Presentación de la dinámica y agrupación}

La experiencia se desarrolló en tres sesiones presenciales con 30 estudiantes de Fundamentos de Teoría de Máquinas y Mecanismos, asignatura troncal del primer cuatrimestre del $2^{\circ}$ curso de Grado de Ingeniería Mecánica. En la primera sesión, se desarrolló la exposición teórica por parte del profesorado, tras lo cual se procedió a la ejemplificación de un problema y su resolución. En la segunda sesión, se repasó el contenido de la primera sesión y se procedió a presentar el ejercicio que el alumnado tenía que solventar. Se planteó con instrucciones claras el trabajo a desarrollar. Se explicaron los criterios para el éxito de la experiencia, comunicándoles claramente qué se esperaba de ellos y cómo se desarrollaría la actividad. Sin duda, identificar el reto al que se enfrentaban los estudiantes les permitía afrontar la tarea más motivados. Además de aclarar la responsabilidad individual, se explicó en qué consistía la cooperación grupal, destacando el valor de la interdependencia positiva (la necesidad del conocimiento de los compañeros para el propio aprendizaje) y se especificaron las conductas deseables en los alumnos para que la actividad se desarrollase adecuadamente y alcanzase los objetivos y el éxito esperados. Se dio gran importancia a que todos los alumnos comprendieran el proceso y asumieran el compromiso y las responsabilidades que se ponían en juego.

En la práctica, el ejercicio fue abordado de manera individual durante 20 minutos, según el tiempo establecido. Posteriormente, siguiendo las directrices establecidas en la planificación previa, se procedió a los emparejamientos (parejas, grupos de cuatro, grupos de ocho, gran grupo) que precisaron más de los 5 minutos programados, optándose a ampliarlos a 15 y 10 minutos. Transcurrido ese tiempo se presentaron al gran grupo los resultados. El profesor evitó expresar opiniones o dar información que facilitase a los estudiantes la respuesta correcta. Esto permitía aprovechar el error como una oportunidad para el aprendizaje, mostrando que el camino recorrido, hasta alcanzar el resultado final, era el verdadero aprendizaje. En este proceso de modelar pensamientos de orden mayor resultó útil hacer preguntas que verificasen el conocimiento profundo de los estudiantes, como podían ser las siguientes: ¿Por qué? ¿Qué significa? ¿Cómo se relaciona... con ...?

\subsection{Fase III: Seguimiento y observación por parte del profesor}

Resultaba fundamental que, en esta labor de seguimiento, el profesorado prestase atención a lo que acontecía en los grupos cooperativos, tanto en los contenidos como en los comportamientos, y tomase notas con el fin de obtener información que volcar en las valoraciones que debía emitir como conclusión de la actividad. Por ello, se elaboró una plantilla (Tabla 1) con unas sencillas pautas de observación para el docente, relacionadas tanto con la capacidad del alumnado para resolver el problema, como para usar habilidades sociales necesarias para cooperar con otros.

Tabla 1.

Plantilla de observación

\begin{tabular}{|c|c|c|c|c|c|}
\hline & $\begin{array}{c}\text { Trabajo } \\
\text { individual }\end{array}$ & Parejas & $\begin{array}{c}\text { Grupos } \\
\text { de } \\
\text { cuatro }\end{array}$ & $\begin{array}{c}\text { Grupos } \\
\text { de } \\
\text { ocho } \\
\end{array}$ & $\begin{array}{l}\text { Gran } \\
\text { grupo }\end{array}$ \\
\hline $\begin{array}{l}\text { Comprensión } \\
\text { del problema } \\
\text { planteado } \\
\text { Resolución de la } \\
\text { tarea } \\
\text { Actitud hacia la } \\
\text { experiencia } \\
\text { Grado de } \\
\text { colaboración }\end{array}$ & & & & & \\
\hline
\end{tabular}

Fuente: Los autores. 
Esas observaciones permitieron al docente intervenir clarificando instrucciones, respondiendo preguntas, enseñando y mejorando destrezas, etc.. Teniendo en cuenta las indicaciones del profesor David Johnson [5], durante esta fase el docente debía animar a los estudiantes a que compartiesen sus conocimientos y sus estrategias de aprendizaje, dentro de un clima de respeto y entendimiento mutuo.

La observación se complementó con un diario de prácticas para reflexionar acerca de todo lo visto, vivido, trabajado. Otras cuestiones no abarcables con la observación y el análisis de documentos escritos elaborados por el alumnado, como captar lo que las personas piensan, perciben, sienten o como interpretan el significado que otorgan a diferentes ámbitos de su experiencia, serían abordadas mediante las conversaciones surgidas durante la experiencia.

\subsection{Fase IV: Evaluación}

La evaluación se inició con la exposición, análisis y resolución de la actividad por parte de los grupos de 8 miembros. Los representantes expusieron los resultados obtenidos al resto de compañeros. Finalizada la ronda de intervenciones, el profesor abrió un diálogo para confirmar los aciertos y aclarar las dudas que los errores plantaban. El papel del docente en esta fase fue incitar a los estudiantes a escuchar la diversidad de opiniones, a desarrollar un pensamiento crítico y creativo y a participar en diálogos abiertos y significativos mediante la emisión de críticas constructivas que permitan una aceptación positiva.

Al terminar el grupo, y como aconseja Alfie Kohn [6], el profesor incentivó una retroalimentación positiva acerca del trabajo realizado. Al hacerlo, no dijo a los estudiantes si estuvo bien o mal, sino que les hizo preguntas que les ayudasen a verbalizar cómo llegaron a la solución presentada. Con esta evaluación, que estimula la participación del alumnado guiado por el profesor, se pretendía lograr estudiantes más autónomos, autoreflexivos y responsables. En este sentido y como recogen Patricia Morales y Victoria Landa [7], el rol del tutor se puede entender mejor en términos de comunicación metacognitiva ya que "el tutor plantea preguntas a los estudiantes que les ayude a cuestionarse y encontrar por ellos mismos la mejor ruta de entendimiento y manejo del problema". Al finalizar la sesión, se entregaron al profesor los trabajos individuales y grupales desarrollados.

Tomando como referencia a Pedro Morales Vallejo [8], docente e investigador en el Departamento de Educación, Métodos de Investigación y Evaluación de la Universidad Pontificia Comillas de Madrid, en la tercera sesión, tras nueva entrega de los trabajos individuales debidamente corregidos, se procedió a registrar una valoración cualitativa por parte del alumnado. Se pidió que, individualmente y de forma anónima, describiesen sus impresiones sobre la experiencia, tanto en lo relacionado con los contenidos trabajados y los conocimientos adquiridos, como los aspectos cooperativos desarrollados. Paralelamente cada estudiante evaluó cuantitativamente la experiencia según los siguientes criterios de valoración (Tabla 2).
Tabla 2.

Rúbrica de evaluación de la experiencia por parte del alumnado

\begin{tabular}{l} 
Criterio \\
El trabajo ha \\
propiciado la \\
responsabilidad \\
individual \\
Los miembros \\
del grupo \\
explicaron \\
unos a otros lo \\
que estaban \\
aprendiendo. \\
Los miembros \\
del grupo \\
participaron en \\
igual medida. \\
El grupo \\
trabajó con \\
eficacia. \\
Se logró la \\
comprensión \\
del problema. \\
El alumnado \\
resolvió el \\
problema \\
planteado de \\
manera \\
satisfactoria. \\
El trabajo fue \\
concluido en el \\
tiempo \\
estipulado. \\
\hline
\end{tabular}

Fuente: Los autores.

\section{Resultados}

\subsection{Con respecto al docente}

Frente a la clase magistral, en la experiencia desarrollada, el docente consideró haber ampliado sus competencias. Al convertirse en gestor favorecedor de ambientes de aprendizaje desempeñó dos roles fundamentales. Por un lado, realizó la labor de diseñador instruccional que facilitaba condiciones en el entorno de aprendizaje para que fuese más efectivo y definió las actividades que se necesitaban afianzar o aprender por parte del grupo de estudiantes.

Más concretamente, el profesor, como diseñador instruccional, se encargó de definir las condiciones iniciales del trabajo: planeó los objetivos académicos y los conocimientos a adquirir, planificó y explicó los criterios de éxito, estableció las tareas a realizar, diseñó materiales, definió los mecanismos de evaluación y monitoreó el aprendizaje de los alumnos en clase, en este sentido la hoja de observación fue útil para orientar la valoración final emitida como cierre de la actividad. Pero su función principal como diseñador instruccional fue crear ambientes de aprendizaje, brindando oportunidades para el trabajo colaborativo y ofreciendo a los estudiantes una variedad de tareas de aprendizaje auténticas. 
Por otro, y coincidiendo con Howard S. Barrows [9], en esta experiencia el papel del docente no se ciñó a transmitir unos conocimientos, sino que se transformó en mediador cognitivo al intentar que el alumnado desarrollase habilidades de razonamiento (resolución de problemas, metacognición, pensamiento crítico) y fuese autónomo de su propio aprendizaje.

Frente a los resultados obtenidos en una clase magistral de la asignatura Fundamentos de Teoría de Máquinas y Mecanismos, los alcanzados en estas clases activas, donde se aplicó una técnica simple de trabajo cooperativo, resultaron de mayor calidad. Esto se debió a que se produjo una mejora que abarcó, no solo un mayor número de estudiantes que alcanzaron una resolución adecuada de los problemas planteados de análisis cinemático; sino que, también, se incentivó la implicación del alumnado, su entusiasmo, su motivación y la conciencia del nivel de aprendizaje adquirido. Ambos aspectos muestran que el profesor alcanzó los objetivos programados sobre la mejora del nivel de formación de los estudiantes y del rendimiento académico en la asignatura de Fundamentos de Teoría de Máquinas y Mecanismos, y sumó la satisfacción propia por una docencia más integradora, al propiciar aprendizajes más complejos e incentivar la motivación del alumnado por la asignatura.

En definitiva, el profesor valoró muy positivamente la incorporación de estrategias cooperativas porque, debidamente planificadas, son fáciles de aplicar en el aula, son compaginables con la clase magistral, se alcanzan resultados muy positivos, y actualiza al docente permitiéndole trabajar por competencias.

\subsection{Con respecto al alumnado}

El alumnado emitió una alta valoración sobre la experiencia, recogida en la Tabla 3. De estas aportaciones se destaca que la actividad permitió que todos participasen; posibilitó alcanzar acuerdos al enfocar sus esfuerzos hacia un fin claro; favoreció la retroalimentación, facilitando que el alumnado detectase y resolviese problemas, dudas y alcanzara un alto rendimiento; ayudó a usar estrategias para asumir la responsabilidad de sus propios aprendizajes al generar mayor compromiso individual y guió en el camino de la cooperación, el beneficio mutuo y la cohesión grupal.

En la evaluación emitida por el alumnado se desveló el carácter innovador del trabajo cooperativo desarrollado y la idoneidad con respecto a las competencias que, del alumnado, demanda la asignatura de FTMM. Se evidenció cómo los estudiantes eran conscientes de carecer de habilidades suficientes de trabajo en equipo. La causa se atribuía a la carencia de prácticas similares, que estuviesen organizadas de manera adecuada para un trabajo en grupo que implicase un enriquecimiento mutuo, que permitiese compartir metas claramente definidas con objetivos conocidos, medibles y autoevaluables $\mathrm{y}$, especialmente, una planificación que favoreciera el autoaprendizaje.

Tabla 3.

Valoración de la experiencia por parte del alumnado

Valoración

cuantitativa

emitida por el Valoración cualitativa emitida por el alumnado alumnado (Rúbrica)

"Mi experiencia es positiva, ya que no tenía muy dominada esta parte de la asignatura y con la colaboración del grupo me ha ayudado bastante a resolver ciertas dudas encontradas en el ejercicio... el alumno se siente más cómodo exponiendo sus dudas ante un compañero que ante un profesor."

"El que un concepto te lo explique un compañero, un igual, puede hacer que lo comprendas mucho más rápido. El aprender a trabajar conjuntamente con personas desconocidas puede ser muy bueno, ya que tienes que adaptarte a las características del grupo."

"Yo tenía algunas dudas de cómo encontrar los CIR y gracias a esta propuesta de trabajo se han solucionado todas."

"Aparte de relacionarnos más con los compañeros, da la oportunidad de que aquellos que sepan hacerlo se lo expliquen a quienes no sepan o estén un poco perdidos en el tema... Personalmente he aprendido bastante en esta sesión ya que no sabía lo que había que hacer y ahora lo he comprendido bastante."

Sobresaliente: "Es una forma diferente de aprender ya que lo que cada 18 estudiantes alumno ha aprendido de forma individual lo aporta a los compañeros... esto permite que quienes saben hacerlo afiancen los conocimientos y los que no, de este modo, sí puedan aprenderlos definitivamente."

"Los que saben pueden compartir su experiencia y adquirir más puntos de vista y los que saben menos pueden mejorar."

"Se consigue que la globalidad de la clase acabe sabiendo hacer el ejercicio... en la clase normal te puedes quedar con dudas que, por vergüenza u otra razón, no preguntas. De este modo, eso no pasa... y a la vez mejoras las relaciones con algunos compañeros."

"Personalmente me ha ayudado en tanto que he aclarado la mayor parte de las dudas y de eso se trataba, de aprender."

"Para poder ayudar hace falta comprender muy bien el problema y eso me ha beneficiado."

"Puntúo con un sobresaliente la actividad basándome en que nos ha servido para aprender, compartir conocimientos y cooperar."

"Me parece buena forma de enterarte de cómo se trabaja en grupo. Por otro lado te das cuentas de errores que ignorabas gracias a la comparación con otros compañeros."

"Me parece una excelente forma de trabajar. Es una experiencia innovadora ya que es la primera vez que la he realizado y los resultados me han parecido muy buenos... Lo único que yo cambiaría sería la cantidad de movimiento en el aula."

Notable: "Esta es una gran forma de aprender. Aunque el tiempo 12 estudiantes en la última agrupación era escaso."

"Me ha parecido buena forma de trabajar, pero como inconveniente le veo que si te agrupas con alguien que tiene resultados muy diferentes, tal vez no se consiga aclarar el tema."

"Avisar con antelación para traer los conocimientos frescos y aprovechar mejor la experiencia."

"Demasiado tiempo organizando los grupos (la próxima vez como están hechos ya no sucederá)."

Fuente: Los autores.

Unido a las valoraciones emitidas por el alumnado, los resultados objetivos fueron contrastables, esencialmente a 
través de las respuestas escritas desarrolladas y de las ideas expuestas durante las interacciones entre el profesor y el alumnado, tanto en grupos como individualmente. Por tanto, la certeza de la profundización del aprendizaje quedó garantizada por la calidad de la participación en el aula y la asimilación de los conceptos ingenieriles expresados. Esto ha sido evidente, especialmente en aquellos alumnos que se perdían en la clase magistral al no expresar sus dudas ya sea por timidez, por falta de fluidez verbal o por lagunas sobre la materia que le impedían seguir el ritmo habitual de la misma.

Además, en las valoraciones del alumnado, tanto cuantitativa como cualitativa, se confirmó que el aprendizaje cooperativo permitía la adquisición de los contenidos, unido al desarrollo de habilidades y estrategias autorreguladoras encaminadas a la resolución de problemas y, especialmente, a "aprender a aprender" y "aprender a pensar". Igualmente se enfatizó la importancia del sentido personal que los alumnos le daban a lo que aprendían. En sus representaciones y expectativas, el alumnado evidenció el papel que desempeñaba en su propio éxito o fracaso y percibió su conexión entre el mismo y las relaciones que se establecían con el profesor y sus compañeros.

Y, principalmente, las opiniones vertidas reflejaban la posibilidad que ofrecía el aprendizaje cooperativo de atribuirle sentido personal a lo que se aprendía. Mariana Miras, profesora del Departamento de Psicología Evolutiva y de la Educación de la Universidad de Barcelona [10], destaca especialmente este mundo de emociones y representaciones, activado en el aula al aprender, ya que determina el autoconcepto académico, la autoestima, el interés latente, los “yoes" posibles, la motivación y el enfoque que el estudiante adopta y las creencias que activa.

Los resultados de la experiencia de innovación desarrollada se alinean con las perspectivas que cuestionan el papel del docente universitario como mero trasmisor e instructor, sistemático y eficiente, de ideas, conocimientos o doctrinas [1114]. El estudio desarrollado nos muestra que los docentes necesitan comprender y asumir la relevancia de las maneras en cómo la enseñanza debe facilitar el compartir y apropiar saberes los unos de los otros. Además, los resultados obtenidos avalan la necesidad de ahondar en la potenciación, el desarrollo y la aplicación de las diferentes capacidades del estudiante mentales, cognitivas, afectivas, socioculturales- ya que su puesta en juego los impulsa a tener, no sólo éxito académico, sino también éxito en su futura vida laboral y en su vida cotidiana.

Los resultados alcanzados nos muestran la importancia de la toma de decisiones que cada profesor hace a diario sobre la definición, planificación y selección de contenidos, tareas, enfoques de enseñanza y sistemas de evaluación. Estas decisiones normalmente se limitan a conseguir que los estudiantes comprendan, analicen y expliquen conceptos. Con ello, se olvida que el docente debe contribuir, también, a que el estudiante realice un aprendizaje significativo facilitando que evalúe contenidos, cree, diseñe y conjeture realidades y utilice, aplique e implemente conocimiento. La práctica de técnicas simples de trabajo cooperativo ha mostrado su eficacia al respecto, siempre que esté sustentada en una adecuada y rigurosa planificación.
Finalmente, contar con una mayor visión educativa ha permitido al docente entender un poco más sus teorías implícitas y las maneras como éstas influyen en su práctica profesional y en el proceso de aprendizaje de sus estudiantes. En consecuencia, se deduce la necesidad que tiene el profesorado de paliar la escasez de conocimientos sobre las dimensiones que facilitan o inhiben el aprendizaje. Esa actualización es necesaria para poder aplicar, en los contextos y prácticas diarias de aula, propuestas adecuadas de aprendizaje que logren formar a los estudiantes en el éxito en y fuera del aula. Y esto solo es posible si esas prácticas responden a las necesidades e intereses de la Educación del siglo XXI, que exige al docente "formar al estudiante para la complejidad y el desconcierto de la contemporaneidad" [12]. En definitiva: "Hay, pues, que prepararse para nuestro mundo incierto y contar con lo inesperado" [13].

\section{Conclusión}

Se ha descrito una situación en la cual se establecían interacciones que conllevaban a mecanismos de aprendizaje. Las condiciones, según la experiencia desarrollada, que han contribuido a que ese aprendizaje ocurriese efectivamente fueron las siguientes:

- Una adecuada planificación con metas y procedimientos claramente definidos.

- Establecer de manera precisa y asumible las funciones y responsabilidades de los participantes.

- Implicar e incentivar que las funciones y tareas a realizar sean aceptadas por el alumnado.

- El riguroso cumplimiento de los tiempos se traduce en un gran dinamismo controlado y productivo. Ser conscientes del límite de tiempo con el que se cuenta, favorece que el alumnado enfoque sus esfuerzos en la resolución del problema.

- Potenciar un clima de colaboración y ayuda mutua.

- Se ha de conseguir una dependencia genuina derivada de la necesidad de compartir el conocimiento en términos explícitos, llegando al entendimiento y la obtención de conclusiones comunes.

La experiencia desarrollada evidencia las fortalezas y debilidades de los aprendizajes individuales y abre posibilidades de superación y mejora de los mismos a través del trabajo cooperativo. Es importante partir del esfuerzo individual hasta llegar a la suma de todos los esfuerzos. El aprendizaje se expande y se hace extensivo a un mayor número de alumnos que el que se produce en una clase tradicional y meramente expositiva. Las interacciones del grupo generan retroalimentación de los conocimientos, además de motivación y satisfacción por el propio aprendizaje.

Esta enseñanza genera aprendizaje productivo al formar alumnos que, de forma activa y no reproductiva, construyen y transforman sus conocimientos. Necesitamos activar la educación tradicional a través del diseño de estrategias metodológicas activas y participativas. La utilización de metodologías activas de aprendizaje, por parte de los docentes, genera un cambio en la orientación formativa que permite a los estudiantes llegar a ser profesionales integrales, reflexivos, 
creativos y, especialmente, les proporciona una sólida base de conocimientos científicos y técnicos, además de capacidades para desenvolverse en escenarios reales.

En definitiva, el cambio docente no ha de ser necesariamente drástico. Estas prácticas son viables y fáciles de abordar, si se realizan con la adecuada planificación y respondan a necesidades reales de aprendizajes. Además, son un complemento adecuado a la clase magistral, que no precisa ser abandonada ni estigmatizada. Lo realmente difícil es cambiar nuestra percepción de qué es y cómo se produce el aprendizaje y nuestro papel como docente.

\section{Bibliografía}

[1] Laboratorio de Innovación Educativa José Ramón Otero, Qué - Por qué - Para qué - Cómo. Aprendizaje cooperativo. Propuesta para la implantación de una estructura de cooperación en el aula. Sociedad Cooperativa Madrileña, Colegio Ártica. 2009.

[2] Dillembourgh, P., What do you mean by Collaborative Learning? In P.Dillenbourgh (Ed) Collaborative-learning: Cognitive and Computational Approaches, pp. 1-19. 1999, Oxford:Elsevier.

[3] Aprendizaje cooperativo. Servicio de Innovación Educativa de la Universidad Politécnica de Madrid. 2008.

[4] Prieto, L., El aprendizaje cooperativo, Madrid: PPC,. 2007.

[5] Johnson, D.W., Johnson, R.T. y Holubec, E.J. El aprendizaje cooperative en el aula. Buenos Aires, Paidos Ibérica. 1999,

[6] Kohn, A., Punished by rewards. New York: Houghton Mifflin. 1993,

[7] Morales, P. y Landa, V., Aprendizaje basado en problemas. Theoria, Vol. 13, 2004, 145 P.

[8] Morales, P., Aprender a trabajar en equipo evaluando el proceso. En, Prieto, L. (coord.). La enseñanza universitaria centrada en el aprendizaje. Barcelona, Octaedro. Pp. 133-151, 2007.

[9] Barrows, H.S., The Tutorial Process. Springfield, IL: Southern Illinois University School of Medicine, 1992.

[10] Miras, M. Afectos, emociones, atribuciones y expectativas: El sentido del aprendizaje escolar, en: Coll, AC., Palacios, J. y Marchesi, A., (Comp.): Desarrollo psicológico y educación, 2. Psicología de la educación escolar. Madrid: Alianza. 2001.

[11] Alcántara, L. y Bernal, S., El aprendizaje autorregulado y su relación con la motivación en estudiantes universitario. Didac, 59, 2011.

[12] De Miguel, M. (Coord.) Metodologías de enseñanza y aprendizaje para el desarrollo de competencias. Orientaciones para el profesorado universitario ante el Espacio Europeo de Educación Superior. Madrid, Alianza Editorial, 2006.

[13] Marcelo, C., Función docente: Nuevas demandas para viejos propósitos, en Marcelo, C. (coord.) La función docente. Madrid, Síntesis. 2001.

[14] Perrenoud, P., La universidad: Entre transmisión de saberes y desarrollo de competencias. Pedagogía y Saberes, 24, pp. 67-77, 2006.

[15] Fandiño, Y.J., Una enseñanza e investigación inteligentes de la inteligencia para el éxito escolar y el éxito en la vida cotidiana. Revista Iberoamericana de Educación 46(9). Ed. Organización de Estados Iberoamericanos para la Educación, la Ciencia y la Cultura (OEI). pp. 1$12,2008$.

[16] Morin, E., La mente bien ordenada. Seix Barral, Los tres Mundos, Ensayo. Barcelona. 2004, 79 P.

J.J. Prieto-Romero, recibió el título de Ing. de Organización Industrial en 2002, y el título de Dr. en Ingeniería en 2009, todos ellos de la Universidad Cádiz, España. Desde 1991 trabaja como Ingeniero Municipal en los Servicios Técnicos Municipales del Excmo. Ayuntamiento de Palos de la Frontera. Se vinculó a la Escuela Superior de Ingeniería de la Universidad de Huelva en el año 2006 como profesor Dr. asociado. Sus intereses investigativos incluyen: Historia y evolución de las maquinas, mecanismos e ingeniería mecánica y su influencia en los sectores productivos, culturas y especialmente en el descubrimiento de América. Metodologías activas en el aula, trabajo colaboratívo y cambios en los modelos educativos como nuevas herramientas de aprendizaje.

ORCID: 0000-0003-0014-1199
M.A. Martín-Maraver, recibe el título Diplomada de EGB en 1986 por la Universidad de Córdoba, España; Experta Universitaria en Convivencia Escolar por la Universidad Nacional de Educación a Distancia, España, en 2004; Licenciada en Humanidades por la Universidad Oberta de Cataluña, España, en 2012. Desde 1987 hasta 2006 trabajó en centros educativos, como funcionaria de carrera, impartiendo el área de lengua castellana y lengua inglesa en el nivel de Educación Secundaria Obligatoria. Desde 2006 trabaja en el Centro del Profesorado Sevilla 6 de Lora del Río, España, ejerciendo actualmente la Vicedirección de dicha institución. Centra su labor investigadora en la formación del profesorado relacionada con la mejora de los procesos de enseñanza y aprendizaje y la educación inclusiva.

ORCID: 0000-0002-4433-8236 